\title{
BLOOD KETONES AND SERUM LIPIDS IN STARVATION AND WATER DEPRIVATION $1,2,3$
}

\author{
By B. L. KARTIN, E. B. MAN, A. W. WINKLER, AND J. P. PETERS \\ (From the Aero-Medical Laboratory, Wright Field, Dayton, Ohio, and the Departments of Internal \\ Medicine and of Psychiatry, Yale University School of Medicine, \\ New Haven)
}

(Received for publication March 27, 1944)

Few observations of the behavior of serum lipids in acute human starvation have been reported and no studies using present improved analytical methods are available. The occasional published reports of changes in serum lipids in fasting experimental animals are often contradictory (1 to 9 ). Increases in the blood ketone bodies during starvation are well recognized (10 to 15), but have never been systematically related to serum lipid changes.

In this study, serum lipids and blood ketones were determined simultaneously in 14 fasting normal male human subjects, some of whom were also deprived of water. These have been supplemented with experiments on fasting mon-

${ }^{1}$ Lt. Col. D. B. Dill, Q. M. C., A. U. S., was responsible for the inception of this work and guided it through its initial stages. Capt. J. M. Quashnock, M. C., A. U. S., assisted in the management of the experiments and in the analysis of the data, as well as serving as a volunteer subject. Capt. Wilson, A. C., A. U. S., Lt. Holmes, Sn. C., A. U. S., Lt. Chovnick, A. C., A. U. S., Sgts. Tressler and Roberts, and Pvts. Perzanowski, Leyzorek, and McHugh, all of the A. C., A. U. S., served as volunteer subjects. We are indebted to Dr. T. S. Danowski, Dr. A. J. Eisenman, Dr. J. R. Elkinton, and Dr. E. A. H. Sims of the Department of Internal Medicine of Yale University School of Medicine, for their cooperation and help. Sugar and protein analyses were done by members of the laboratory staffs at Wright Field and at Yale. Miss Barbara Russell of the Yale University School of Nursing and Dr. S. C. Harvey of the Department of Surgery provided proper facilities for the care of the subjects. The work was carried out in connection with an Army Air Corps Contract.

The authors are indebted to Professor John Fulton who generously placed the facilities of the Laboratory of Physiology of the Yale University School of Medicine at their disposal.

2 This article has been cleared for publication by the War Department Bureau of Public Relations. The opinions expressed are those of the authors and do not necessarily reflect official views of the War Department.

- The ketone investigation was aided by a grant from the Dazian Foundation for Medical Research. keys and dogs. Some effects of feeding carbohydrate or fish to otherwise fasting subjects have also been included.

\section{EXPERIMENTAL PROCEDURE}

Fourteen normal adult male subjects were starved for periods of 2 to 6 days. Three of them, Yale medical students, were subjected only to a 2-day fast and were allowed water or black coffee ad libitum. The other 11 volunteers, members of the Army of the United States, were subjected to water restriction or deprivation. Some of them were subjects of carbohydrate and of fish feeding experiments as well. The organization of these experiments has been described in detail in an accompanying paper (16), and is summarized in Table I of that paper. Serum proteins were not determined in the medical students and serum lipids were not determined in the subjects eating fish. Intravenous glucose tolerance tests (17) with simultaneous blood ketone determinations were made at the end of 8 starvation periods in Experiments II and III and blood ketones were repeated in 3 of them after 24 hours of carbohydrate ingestion. After 2 periods of fasting, blood ketones were determined before and after 24 hours of sugar eating. Intravenous glucose tolerance tests were also carried out at the end of 4 experiments with fish feeding.

Four male rhesus monkeys (macaca mulatta) and 7 dogs were studied. The monkeys were starved for 3 to 6 days, the dogs for longer periods. All animals, with the exception of 2 dogs, were permitted to drink water ad libitum. In other experiments, the monkeys were allowed to eat varying amounts of sugar for 3 to 8 days.

\section{CHEMICAL METHODS}

Serum separated from clotted venous blood drawn under oil was used for all lipid analyses, while oxalated whole blood was used for the ketone determinations. In Experiment I, most of the analyses were carried out on blood or serum which had been transported for about 24 hours in special cooled containers. This procedure was justified by control experiments in which determinations of serum lipids and blood ketones in blood samples precipitated immediately were compared with those in blood samples precipitated after $\mathbf{4 8}$ hours of refrigeration. No significant change was found. The extra manipulations and transportation prevented duplicate analyses in a few instances and may have been responsible for some inconsistencies in the data of Experiment I. 
Serum lipids, proteins, and blood sugars were determined by methods previously described (17 to 23). Neutral fat concentration was calculated by the formula of Peters and Man (24). The values for neutral fat calculated in this way are less accurate than are the values for the other lipid fractions, since the calculation introduces cumulative errors from 3 chemical methods and from variations in composition of phospholipids. Nevertheless, in each of 3 normal males, the greatest individual deviation from the average neutral fat of that individual was only $\pm 0.8 \mathrm{~m}$.eq. per liter $(24,25)$. Whole blood ketones were determined by the method of Weichselbaum and Somogyi (26), with a combination of the procedures used in deproteinization and desaccharification. Results are expressed as mgm. per cent of acetone. At concentrations below $1 \mathrm{mgm}$. per cent, the proportion of known amounts of acetone recovered from blood was considerably less than that reported by the original authors. This is not a serious practical difficulty, however, since in this range a large percentage error represents only a small absolute one. At higher concentrations, the proportion recovered was similar to that reported by Weichselbaum and Somogyi (26). Nitrogen, water, and electrolyte balances were also measured in the military subjects and are reported elsewhere (16).

\section{RESULTS}

\section{A. Normal human subjects}

The data from all fasting normal subjects are contained in Table I. Subject $T$ was atypical throughout, in that his carbohydrate tolerance, urinary nitrogen excretion, blood ketones, and serum lipids were little affected by starvation. Subjects $\mathrm{D}$ and $\mathrm{H}$ drank large amounts of dilute salt water on the fifth day of Experiment I, thereby certainly affecting the concentration of serum lipids at the close of the period. Data from all experiments with $\mathrm{T}$ and from these experiments with $\mathrm{D}$ and $\mathrm{H}$ are therefore considered separately, and the general statements made below concerning the results do not apply to them. In analyzing the data concerning lipids and proteins of serum, each change is referred to the initial concentration in the blood of the individual in the experiment under consideration.

Total cholesterol increased progressively in starvation. At the end of 2 days, the average rise did not usually exceed the average variation observed in normal subjects, $\pm 13 \mathrm{mgm}$. per cent (derivation of normal average variations of cholesterol and lipid phosphorus is given under Figure 1). After 3 to 6 days, however, it amounted to $36 \pm 13 \mathrm{mgm}$. per cent, a highly significant increment. This is illustrated in

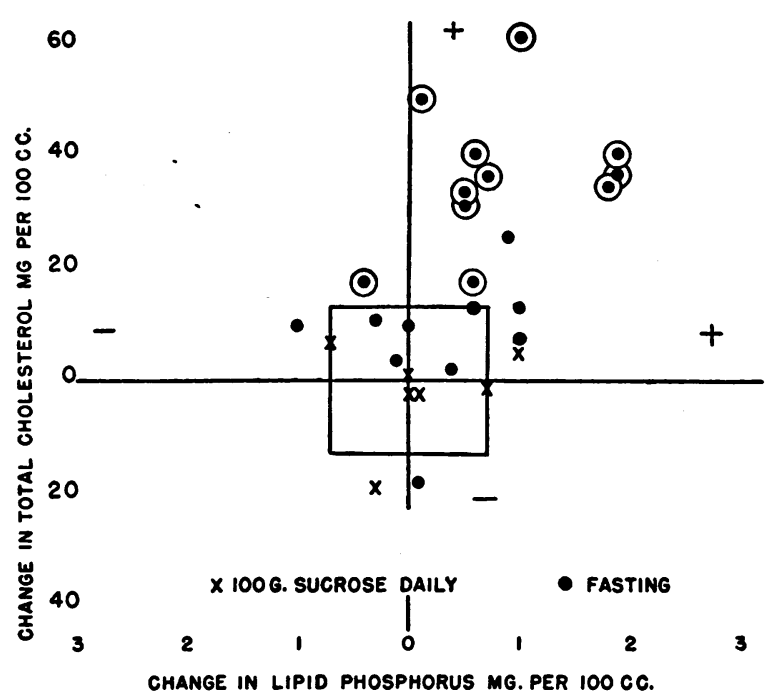

Fig. 1. The Relationship of Changes in Serum Total Cholesterol to the Changes IN Lipid Phosphorus

Dots represent the changes in fasting human subjects, crosses the changes in humans who ingested 100 grams of sugar daily. Circled dots denote values after 3 or more days of starvation. The square in the center denotes the average individual variation for the respective lipid fractions.

The probable error of the averages of duplicate total cholesterol determinations is $5.42 \mathrm{mgm}$. per cent (25). However, the variation in the individual from day to day is probably larger and would include the error of the method. In our own experimental work on 3 healthy male adults, in each subject, the average deviation from his average value did not exceed $\pm 13 \mathrm{mgm}$. per cent of serum total cholesterol $(24,25)$. Sperry (27), in 25 normal adults, found the variation of the individual from the average not to exceed $\pm 12.3 \mathrm{mgm}$. per cent, a value in close agreement with our value of $\pm 13 \mathrm{mgm}$. per cent.

In the same 3 normal males, the greatest average deviation from the average lipid phosphorus of any one individual was $\pm 0.7 \mathrm{mgm}$. per cent $(24,25)$. This includes the error of the method of $\pm 0.13 \mathrm{mgm}$. per cent (25).

Figure 1 in which the 11 observations after 3 or more days of starvation are distinguished by circled dots. In addition, it will be noted in this figure that cholesterol increased to some extent in all but 1 of 21 determinations, irrespective of the duration of starvation. The increase affected both free and esterified fractions of cholesterol. Although in the experiments of 3 or more days, the average increment of esterified cholesterol, $24 \pm 11 \mathrm{mgm}$. per cent, is the greater, the average increase of free choesterol, $13 \pm 3.6$ 
TABLE I

Serum lipids and blood ketones of human subjects

\begin{tabular}{|c|c|c|c|c|c|c|c|c|c|c|}
\hline \multirow{3}{*}{ Subject } & \multirow{3}{*}{ Days } & \multicolumn{5}{|c|}{ Serum lipids } & \multirow{3}{*}{$\begin{array}{l}\text { Ketones } \\
\text { as } \\
\text { acetone }\end{array}$} & \multirow{3}{*}{$\begin{array}{c}\text { Total } \\
\text { proteins }\end{array}$} & \multirow{3}{*}{ Food**** } & \multirow{3}{*}{ Fluid***** } \\
\hline & & \multicolumn{2}{|c|}{ Cholesterol } & \multirow{2}{*}{$\begin{array}{c}\text { Lipid } \\
\text { phosphorus }\end{array}$} & \multicolumn{2}{|c|}{ Fatty acids } & & & & \\
\hline & & Total & Free & & Total & $\begin{array}{c}\text { Neutral } \\
\text { fat }\end{array}$ & & & & \\
\hline & & $\begin{array}{l}\text { mgm. per } \\
100 \text { cc. }\end{array}$ & $\begin{array}{l}\text { mgm. per } \\
100 \mathrm{cc} .\end{array}$ & $\begin{array}{l}\text { mgm. per } \\
100 \mathrm{cc} .\end{array}$ & $\begin{array}{c}\text { m. eq. per } \\
1000 \mathrm{cc} \text {. }\end{array}$ & $\begin{array}{l}\text { m. eq. per } \\
1000 \text { cc. }\end{array}$ & $\begin{array}{l}\text { mgm. per } \\
100 \text { cc. } \\
\text { whole blood }\end{array}$ & $\begin{array}{l}\text { grams per } \\
100 \text { cc. } \\
\text { serum }\end{array}$ & & \\
\hline
\end{tabular}

Experiment I

\begin{tabular}{|c|c|c|c|c|c|c|c|c|c|c|}
\hline $\mathbf{L}$ & $\begin{array}{l}0 \\
2 \\
5\end{array}$ & $\begin{array}{l}206 \\
210 \\
219\end{array}$ & $\begin{array}{l}50 \\
52 \\
63\end{array}$ & $\begin{array}{l}8.9 \\
8.8 \\
9.5\end{array}$ & $\begin{array}{l}11.8 \\
11.6 \\
11.6\end{array}$ & $\begin{array}{l}2.6 \\
2.4 \\
2.0\end{array}$ & $\begin{array}{r}0.3 \\
8.9 \\
13.9\end{array}$ & $\begin{array}{l}6.79 \\
7.32 \\
7.53\end{array}$ & $\begin{array}{l}0 \\
0\end{array}$ & $\begin{array}{l}\mathbf{R} \\
\mathbf{R}\end{array}$ \\
\hline $\mathbf{F}$ & $\begin{array}{l}0 \\
2 \\
5\end{array}$ & $\begin{array}{l}147 \\
129 \\
209\end{array}$ & $\begin{array}{l}43 \\
44 \\
58\end{array}$ & $\begin{array}{l}8.5 \\
8.6 \\
9.5\end{array}$ & $\begin{array}{l}7.1^{*} \\
8.3^{*} \\
9.8\end{array}$ & $\begin{array}{c}-0.5^{*} \\
1.1^{*} \\
0.4\end{array}$ & $\begin{array}{c}0.3 \\
7.2^{* *} \\
14.8\end{array}$ & $\begin{array}{l}6.56 \\
6.90 \\
7.34\end{array}$ & $\begin{array}{l}0 \\
0\end{array}$ & $\begin{array}{l}\mathbf{R} \\
\mathbf{R}\end{array}$ \\
\hline $\mathbf{Q}$ & $\begin{array}{l}0 \\
2 \\
5\end{array}$ & $\begin{array}{l}185 \\
203 \\
203\end{array}$ & $\begin{array}{l}49 \\
67 \\
56\end{array}$ & $\begin{array}{l}8.7 \\
9.3 \\
8.3\end{array}$ & $\begin{array}{r}10.5 \\
9.6 \\
9.3\end{array}$ & $\begin{array}{l}1.9 \\
0.7 \\
0.7\end{array}$ & $\begin{array}{l}0.4 \\
0.3 \\
2.5\end{array}$ & $\begin{array}{l}7.01 \\
7.40 \\
7.61\end{array}$ & $\begin{array}{l}\mathbf{O} \\
\mathbf{O}\end{array}$ & $\stackrel{\text { O }}{\text { A. L. }}$ \\
\hline D & $\begin{array}{l}0 \\
2 \\
5\end{array}$ & $\begin{array}{l}172^{*} \\
174 \\
171\end{array}$ & $\begin{array}{l}42 \\
56 \\
48\end{array}$ & $\begin{array}{l}7.6 \\
8.0 \\
8.2\end{array}$ & $\begin{array}{c}12.5^{*} \\
8.9 \\
11.7\end{array}$ & $\begin{array}{l}4.7^{*} \\
1.2 \\
3.7\end{array}$ & $\begin{array}{c}0.3 \\
16.6^{* *} \\
23.9^{* *}\end{array}$ & $\begin{array}{l}7.10 \\
7.49 \\
7.30\end{array}$ & $\begin{array}{l}0 \\
0\end{array}$ & $\begin{array}{c}\text { R } \\
\text { D.S. }\end{array}$ \\
\hline $\mathbf{P}$ & $\begin{array}{l}0 \\
2 \\
5\end{array}$ & $\begin{array}{l}192 \\
190 \\
193\end{array}$ & $\begin{array}{l}61 \\
63 \\
47\end{array}$ & $\begin{array}{l}8.9 \\
9.0 \\
8.9\end{array}$ & $\begin{array}{l}12.6 \\
11.7 \\
10.8\end{array}$ & $\begin{array}{l}4.0 \\
3.2 \\
1.9\end{array}$ & $\begin{array}{l}0.3 \\
2.3 \\
3.0\end{array}$ & $\begin{array}{l}6.79 \\
7.48 \\
7.41\end{array}$ & $\begin{array}{l}\text { C } \\
\text { C }\end{array}$ & $\begin{array}{l}\mathbf{R} \\
\mathbf{R}\end{array}$ \\
\hline $\mathbf{R}$ & $\begin{array}{l}0 \\
2 \\
5\end{array}$ & $\begin{array}{l}141^{*} \\
140 \\
146\end{array}$ & $\begin{array}{l}33 \\
46 \\
42\end{array}$ & $\begin{array}{l}6.5 \\
7.2 \\
7.5\end{array}$ & $\begin{array}{l}6.2^{*} \\
8.0 \\
8.3\end{array}$ & $\begin{array}{c}-0.4^{*} \\
1.4 \\
1.2\end{array}$ & $\begin{array}{l}0.3 \\
2.5 \\
3.2\end{array}$ & $\begin{array}{l}6.38 \\
7.03 \\
7.46\end{array}$ & $\begin{array}{l}\mathrm{C} \\
\mathrm{C}\end{array}$ & $\begin{array}{l}\mathbf{R} \\
\mathbf{R}\end{array}$ \\
\hline W & $\begin{array}{l}0 \\
2 \\
5\end{array}$ & $\begin{array}{l}170 \\
177^{*} \\
151\end{array}$ & $\begin{array}{l}52 \\
47 \\
40\end{array}$ & $\begin{array}{l}9.1 \\
8.4 \\
8.8\end{array}$ & $\begin{array}{l}9.7 \\
9.7 \\
8.6\end{array}$ & $\begin{array}{l}1.4 \\
1.5^{*} \\
0.6\end{array}$ & $\begin{array}{l}1.1 \\
1.9 \\
3.0\end{array}$ & $\begin{array}{l}6.63 \\
7.28 \\
7.10\end{array}$ & $\stackrel{\mathrm{C}}{\mathrm{C}}$ & $\begin{array}{l}\mathbf{R} \\
\mathbf{R}\end{array}$ \\
\hline $\mathbf{H}$ & $\begin{array}{l}0 \\
2 \\
5\end{array}$ & $\begin{array}{l}215^{*} \\
213 \\
165\end{array}$ & $\begin{array}{l}56 \\
60 \\
47\end{array}$ & $\begin{array}{l}8.5 \\
8.5 \\
7.6\end{array}$ & $\begin{array}{c}11.4^{*} \\
10.0 \\
8.1\end{array}$ & $\begin{array}{l}2.3^{*} \\
1.1 \\
0.6\end{array}$ & $\begin{array}{l}0.3 \\
2.5 \\
3.4\end{array}$ & $\begin{array}{l}6.97 \\
7.37 \\
6.70\end{array}$ & $\begin{array}{l}\text { C } \\
\text { C }\end{array}$ & $\begin{array}{c}\text { R } \\
\text { D.S. }\end{array}$ \\
\hline
\end{tabular}

Experiment II

\begin{tabular}{|c|c|c|c|c|c|c|c|c|c|c|}
\hline A, $Q$ & $\begin{array}{l}0 \\
2 \\
3 \\
4\end{array}$ & $\begin{array}{l}205 \\
246\end{array}$ & $\begin{array}{l}57 \\
76\end{array}$ & $\begin{array}{l}10.4 \\
11.0\end{array}$ & $\begin{array}{l}11.6 \\
15.8\end{array}$ & $\begin{array}{l}1.7 \\
4.9\end{array}$ & $\begin{array}{r}0.2 \\
16.6 \\
15.5 \\
3.1\end{array}$ & $\begin{array}{l}7.33 \\
8.09\end{array}$ & $\begin{array}{l}\mathrm{O} \\
\mathrm{O} \\
\mathrm{C}\end{array}$ & $\begin{array}{c}\text { O } \\
\text { O } \\
\text { A. L. }\end{array}$ \\
\hline B, Q & 3 & & & & & & 0.6 & & F, C & A.F.J. \\
\hline$A, C$ & $\begin{array}{l}0 \\
2 \\
3 \\
4\end{array}$ & $\begin{array}{r}163 \\
197\end{array}$ & $\begin{array}{l}46 \\
56\end{array}$ & $\begin{array}{l}8.5 \\
9.0\end{array}$ & $\begin{array}{r}9.3 \\
11.3\end{array}$ & $\begin{array}{l}1.3 \\
2.4\end{array}$ & $\begin{array}{r}1.1 \\
12.6 \\
9.8 \\
3.2\end{array}$ & $\begin{array}{l}6.38 \\
7.35\end{array}$ & $\begin{array}{l}\mathbf{O} \\
\mathrm{O} \\
\mathrm{C}\end{array}$ & $\begin{array}{c}\text { O } \\
\text { O. L. }\end{array}$ \\
\hline $\mathrm{B}, \mathrm{C}$ & 3 & & & & & & 6.1 & & $\mathbf{F}$ & A. F. J. \\
\hline A, $\mathrm{H}$ & $\begin{array}{l}0 \\
3 \\
4\end{array}$ & $\begin{array}{l}218 \\
255\end{array}$ & $\begin{array}{l}55 \\
67\end{array}$ & $\begin{array}{l}8.7 \\
9.4\end{array}$ & $\begin{array}{r}9.9 \\
12.7\end{array}$ & $\begin{array}{l}0.6 \\
2.4\end{array}$ & $\begin{array}{r}0.3 \\
13.9 \\
2.4\end{array}$ & $\begin{array}{l}6.99 \\
7.74\end{array}$ & $\stackrel{\text { O }}{\text { C }}$ & $\stackrel{\text { O }}{\text { A. L. }}$ \\
\hline B, H & 3 & & & & & & 7.8 & & $F$ & A. F.J. \\
\hline
\end{tabular}


TABLE I-Continued

\begin{tabular}{|c|c|c|c|c|c|c|c|c|c|c|}
\hline \multirow{3}{*}{ Subject } & \multirow{3}{*}{ Days } & \multicolumn{5}{|c|}{ Serum lipids } & \multirow{3}{*}{$\begin{array}{c}\text { Ketones } \\
\text { as } \\
\text { acetone }\end{array}$} & \multirow{3}{*}{$\begin{array}{c}\text { Total } \\
\text { proteins }\end{array}$} & \multirow{3}{*}{ - Food $* *$} & \multirow{3}{*}{ Fluid } \\
\hline & & \multicolumn{2}{|c|}{ Cholesterol } & \multirow{2}{*}{$\begin{array}{c}\text { Lipid } \\
\text { phosphorus }\end{array}$} & \multicolumn{2}{|c|}{ Fatty acids } & & & & \\
\hline & & Total & Free & & Total & $\underset{\text { fat }}{\text { Neutral }}$ & & & & \\
\hline
\end{tabular}

Experiment II-Continued

\begin{tabular}{|c|c|c|c|c|c|c|c|c|c|c|}
\hline$A, T$ & $\begin{array}{l}0 \\
3 \\
4\end{array}$ & $\begin{array}{l}172 \\
185\end{array}$ & $\begin{array}{l}43 \\
55\end{array}$ & $\begin{array}{l}9.6 \\
9.6\end{array}$ & $\begin{array}{l}10.1 \\
12.7\end{array}$ & $\begin{array}{l}1.2 \\
3.8\end{array}$ & $\begin{array}{l}0.3 \\
3.7 \\
0.9\end{array}$ & $\begin{array}{l}7.32 \\
7.88\end{array}$ & 0 & 0 \\
\hline B, T & $\begin{array}{l}0 \\
3\end{array}$ & $\begin{array}{l}156 \\
149\end{array}$ & $\begin{array}{l}44 \\
39\end{array}$ & $\begin{array}{l}9.2 \\
8.4\end{array}$ & $\begin{array}{l}11.3 \\
11.6\end{array}$ & $\begin{array}{l}3.1 \\
3.9\end{array}$ & 4.7 & $\begin{array}{l}6.68 \\
7.68\end{array}$ & O & A.F.J. \\
\hline A, $\mathbf{R}$ & $\begin{array}{l}0 \\
3\end{array}$ & $\begin{array}{l}117 \\
168\end{array}$ & $\begin{array}{l}32 \\
49\end{array}$ & $\begin{array}{l}7.9 \\
8.0\end{array}$ & $\begin{array}{r}8.1 \\
14.3\end{array}$ & $\begin{array}{l}1.3 \\
6.6\end{array}$ & $\begin{array}{r}0.2 \\
20.8\end{array}$ & $\begin{array}{l}6.28 \\
6.93\end{array}$ & $\mathbf{0}$ & O \\
\hline B, $\mathbf{R}$ & $\begin{array}{l}0 \\
3\end{array}$ & $\begin{array}{l}141 \\
173\end{array}$ & $\begin{array}{l}39 \\
46\end{array}$ & $\begin{array}{l}7.8 \\
8.3\end{array}$ & $\begin{array}{r}8.8 \\
11.9\end{array}$ & $\begin{array}{l}1.7 \\
3.1\end{array}$ & 37.4 & $\begin{array}{l}6.22 \\
6.85\end{array}$ & $\mathbf{O}$ & $0.6 \% \mathrm{NaCl}$ \\
\hline
\end{tabular}

Experiment III

\begin{tabular}{|c|c|c|c|c|c|c|c|c|c|c|}
\hline $\mathbf{H}$ & $\begin{array}{l}0 \\
2 \\
4 \\
5 \\
6 \\
7\end{array}$ & $\begin{array}{l}213 \\
223\end{array}$ & $\begin{array}{l}52 \\
55\end{array}$ & $\begin{array}{l}8.2 \\
8.2\end{array}$ & $\begin{array}{l}10.8 \\
11.2\end{array}$ & $\begin{array}{l}1.8 \\
2.1\end{array}$ & $\begin{array}{r}0.3 \\
17.6 \\
4.5 \\
3.9 \\
2.9 \\
5.0\end{array}$ & $\begin{array}{l}7.12 \\
7.31\end{array}$ & $\begin{array}{l}\text { O } \\
\text { F } \\
\text { F } \\
\text { F } \\
\text { C }\end{array}$ & $\begin{array}{c}\text { R } \\
\text { O } \\
\text { O } \\
\text { O } \\
\text { A. L. }\end{array}$ \\
\hline C & $\begin{array}{l}0 \\
2 \\
4 \\
5 \\
6 \\
7\end{array}$ & $\begin{array}{l}195 \\
203 \\
230 \\
232 \\
236 \\
202\end{array}$ & $\begin{array}{l}52 \\
55^{*} \\
64 \\
66 \\
65 \\
56\end{array}$ & $\begin{array}{c}8.2 \\
9.2^{*} \\
10.0 \\
10.1 \\
10.1 \\
9.1\end{array}$ & $\begin{array}{r}9.4 \\
10.9 \\
11.6 \\
12.2 \\
12.6 \\
11.3\end{array}$ & $\begin{array}{l}1.0 \\
1.7^{*} \\
1.5 \\
2.0 \\
2.4 \\
2.2\end{array}$ & $\begin{array}{r}0.6 \\
11.9 \\
17.7 \\
22.4 \\
33.0 \\
20.5\end{array}$ & $\begin{array}{l}7.07 \\
7.31 \\
7.79 \\
7.82 \\
7.70 \\
7.26\end{array}$ & $\begin{array}{l}0 \\
0 \\
0 \\
0 \\
\text { C }\end{array}$ & $\begin{array}{c}\text { R } \\
\mathbf{O} \\
\mathbf{O} \\
\mathbf{O} \\
\text { A. L. }\end{array}$ \\
\hline $\mathbf{T}$ & $\begin{array}{l}0 \\
2 \\
4 \\
5\end{array}$ & $\begin{array}{l}200 \\
196 \\
200 \\
203\end{array}$ & $\begin{array}{l}\mathbf{5 5} \\
54 \\
54 \\
55\end{array}$ & $\begin{array}{c}11.7 \\
10.9 \\
10.0 \\
9.7^{*}\end{array}$ & $\begin{array}{l}15.7 \\
13.3 \\
14.2 \\
14.1\end{array}$ & $\begin{array}{l}5.2 \\
3.3 \\
4.6 \\
4.6^{*}\end{array}$ & $\begin{array}{l}0.3 \\
3.0 \\
6.5 \\
6.6\end{array}$ & $\begin{array}{l}7.23 \\
7.49 \\
7.98 \\
7.76\end{array}$ & $\begin{array}{l}\mathbf{O} \\
\mathbf{O} \\
\mathbf{O}\end{array}$ & $\begin{array}{c}\mathbf{R} \\
\mathbf{O} \\
\text { A. L. }\end{array}$ \\
\hline $\mathbf{M}$ & $\begin{array}{l}0 \\
2 \\
4 \\
5\end{array}$ & $\begin{array}{l}189 \\
199\end{array}$ & $\begin{array}{l}47 \\
48\end{array}$ & $\begin{array}{r}10.4 \\
9.4\end{array}$ & $\begin{array}{l}13.3 \\
13.0\end{array}$ & $\begin{array}{l}3.6 \\
3.6\end{array}$ & $\begin{array}{r}0.3 \\
9.0 \\
11.1 \\
25.8\end{array}$ & $\begin{array}{l}7.11 \\
7.20\end{array}$ & $\begin{array}{l}\mathrm{O} \\
\mathrm{F} \\
\mathrm{O}\end{array}$ & $\begin{array}{l}\text { R } \\
\text { A. L. } \\
\text { A. L. }\end{array}$ \\
\hline
\end{tabular}

Experiment IV (Students)

\begin{tabular}{|c|c|c|c|c|c|c|c|c|c|}
\hline B & $\begin{array}{l}0 \\
2\end{array}$ & $\begin{array}{l}211 \\
224\end{array}$ & $\begin{array}{l}50 \\
63\end{array}$ & $\begin{array}{l}8.8 \\
9.8\end{array}$ & $\begin{array}{l}11.6 \\
15.6\end{array}$ & $\begin{array}{l}2.3 \\
6.4\end{array}$ & $\begin{array}{r}0.4 \\
29.6\end{array}$ & 0 & A. L. \\
\hline B1 & $\begin{array}{l}\mathbf{0} \\
2\end{array}$ & $\begin{array}{l}232 \\
243\end{array}$ & $\begin{array}{l}60 \\
62 \\
\end{array}$ & $\begin{array}{l}10.5 \\
10.2\end{array}$ & $\begin{array}{l}18.2 \\
17.0\end{array}$ & $\begin{array}{l}7.6 \\
6.4 \\
\end{array}$ & $\begin{array}{r}0.2 \\
12.5\end{array}$ & 0 & A. L. \\
\hline F & $\begin{array}{l}0 \\
1 \\
2\end{array}$ & $\begin{array}{l}163 \\
189\end{array}$ & $\begin{array}{l}48 \\
60\end{array}$ & $\begin{array}{l}6.7 \\
7.6\end{array}$ & $\begin{array}{r}7.8 \\
10.5\end{array}$ & $\begin{array}{l}0.9 \\
2.8\end{array}$ & $\begin{array}{r}0.3 \\
3.9 \\
13.7\end{array}$ & $\begin{array}{l}\text { O } \\
\text { O }\end{array}$ & A. L. \\
\hline
\end{tabular}

* Single determination. All others average of duplicate determinations.

* Poor checks between duplicates.

*** In the food column, $C$ stands for carbohydrate, $F$ for fish in the previous period.

**** In the fluid column, $\mathbf{R}$ stands for restricted and $A$. L. for ad libitum water intake, D. S. for dilute seawater, and A. F. J. for artificial fish juice. 


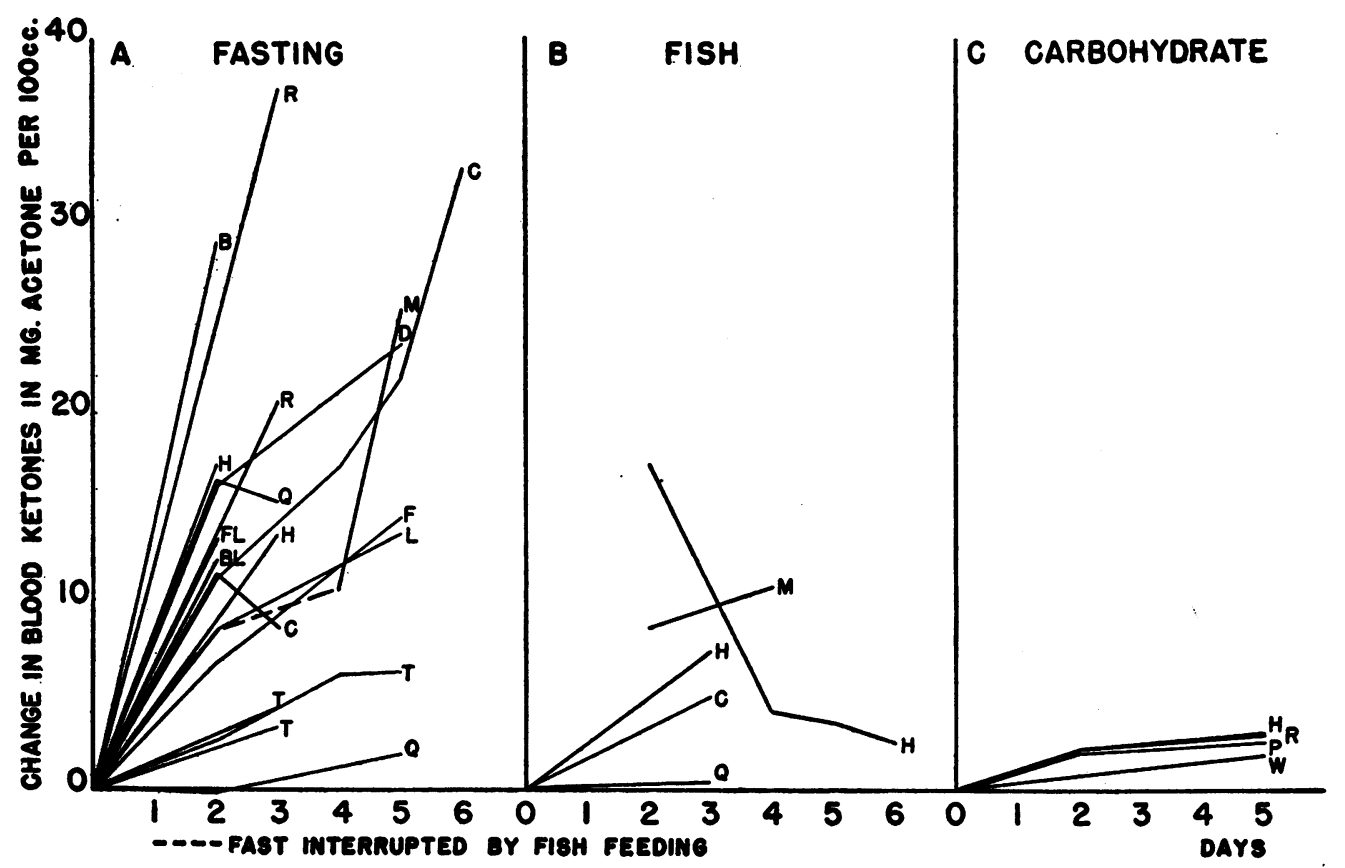

Fig. 2. Blood Ketone Changes in Human Subjects

Fig. 2A. During Starvation

The interrupted line indicates that the starvation was interrupted in one subject by feeding of fish for 2 days.

Fig. 2B. DURing FeEding of Fish

In the 2 instances in which there are no initial values, the subject has been fasted for 2 days prior to the feeding of fish.

Fig. 2C. During Carbohydrate Ingestion

mgm. per cent, is relatively larger and more significant. 4

Lipid phosphorus rose in all but 5 instances, exceeding the range of normal variability, \pm 0.7 mgm. per cent, on 7 occasions (see Figure 1). The average increase after 3 or more days was $0.8 \pm 0.7 \mathrm{mgm}$. per cent.

The average increase of total fatty acids was $1.5 \pm 2.2 \mathrm{~m}$. eq. per liter; for periods of 3 days or more, it was $2.5 \pm 1.9 \mathrm{~m}$. eq. per liter. Changes of neutral fat were quite irregular. In several instances, it decreased. Only at the 3-day interval was it consistently elevated. In all of 5 determinations at this interval, neutral fat was definitely above its initial concentration.

\footnotetext{
- The normal average variation of free cholesterol has been calculated from Sperry's data (28). Free cholesterol was determined on 9 separate occasions in one subject and on 7 occasions in another. Some specimens were taken in the absorptive and others in the post-absorptive state. The average variation of one subject from his own average was $3 \mathrm{mgm}$. per cent, of the other $4 \mathrm{mgm}$. per cent.
}

Whole blood ketone bodies rose consistently and usually progressively during starvation (see Figure 2A). The average initial concentration in the 14 subjects was $0.4 \mathrm{mgm}$. per cent of acetone; after 2 days, it was $11.1 \mathrm{mgm}$. per cent. In most longer studies, it rose further from the second to the fifth day, reaching $37.4 \mathrm{mgm}$. per cent in Experiment II B-R after 3 days and 33.0 mgm. per cent in Experiment III $\mathrm{C}$ after 6 days. In no instance, however, did ketosis attain sufficient severity to produce a significant bicarbonate deficiency (16).

The degree of ketonemia varied from subject to subject and in different experiments on the same subject. There was no exact correlation between ketonemia and hyperlipemia. For example, the greatest ketonemia, $37.4 \mathrm{mgm}$. per cent, occurred in Experiment II B-R, in which cholesterol rose only $32 \mathrm{mgm}$. per cent; whereas a cholesterol increment of $41 \mathrm{mgm}$. per cent in Experiment II A-Q was associated with a blood 
ketone of only $15.5 \mathrm{mgm}$. per cent. Nevertheless, a rough relation between the two functions is implicit in the fact that both rose progressively as starvation was prolonged.

None of the lipid fractions of the serum, except the lipid phosphorus of $R$, rose definitely in the 4 experiments in which subjects took 100 grams of sucrose daily (Experiment I, P, R, W, and $\mathrm{H}$ ). In these experiments, blood ketones did not exceed $3.4 \mathrm{mgm}$. per cent, even after 5 days without other food (Figure $2 \mathrm{C}$ ), although 2 of the same subjects, $\mathrm{H}$ and $\mathrm{R}$ exhibited considerable ketonemia and increases of serum total cholesterol on starvation.

The intravenous injection of 25 grams of glucose at the close of starvation regularly and rapidly reduced the ketonemia of subjects who had starved or received fish (Figures $3 \mathrm{~A}$ and $4 \mathrm{~A}$ ). Subsequent ingestion of carbohydrate during the remainder of the first 24 hours after starvation further depressed blood ketones, although they did not return to normal.

In 3 experiments in which fish was eaten for 3 days (Experiment II $B-Q, C$, and $B$ ), there was less ketonemia than in the comparable control experiments (II $A-Q, C$ and $H$ (see Figure 2B)). Of the 2 subjects who ate fish after a preliminary 2-day fast, only 1 (Experiment III-H) was able to eat considerable quantities. The ketones in this case diminished considerably, but did not return to normal.

Glucose tolerance of all subjects, except $T$, was distinctly low after 3 to 6 days of complete starvation, but was normal in subjects who had

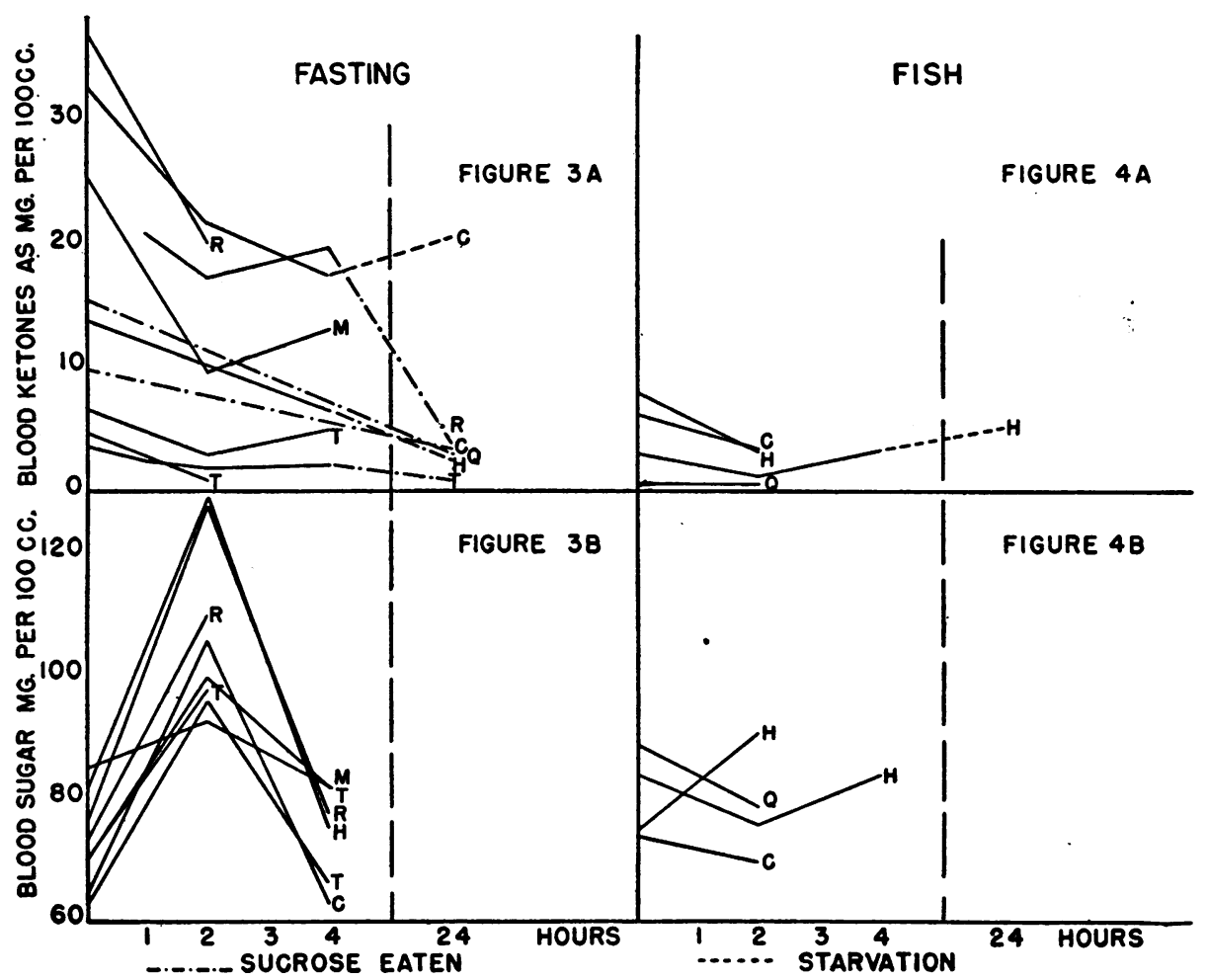

Figs. 3 and 4. Changes in (A) Blood Ketones and (B) Blood Sugar Values, During and Following Intravenous Glucose and Feeding of Sucrose

Fig. 3. Following Starvation

Fig. 4. Following Experiments in Which Fish Had Been Eaten

Solid lines indicate changes following the intravenous injection of 25 grams of glucose at zero time. Dash line extensions indicate continued starvation during the next 24 hours. Dot-dash lines refer to experiments in which 100 to 150 grams of sucrose were ingested at intervals during the period indicated. 
eaten fish (Figures 3B and 4B). No blood sugar below $64 \mathrm{mgm}$. per cent was found in any subject, even after 5 or 6 days of fasting.

\section{B. Experiments with monkeys}

Data from these experiments are given in Table II. Fasting invariably provoked striking hyperlipemia and ketonemia. The serum lipids were elevated at the end of 2 days and rose further subsequently. Cholesterol was most af- fected, increasing on the average $58 \mathrm{mgm}$. per cent, or 49 per cent of the initial value. The increment consisted chiefly of cholesterol esters; consequently, the ratio of free to total cholesterol fell. Lipid phosphorus rose proportionally less than cholesterol, an average of $2.4 \mathrm{mgm}$. per cent, or $\mathbf{3 0}$ per cent of the initial value. Neutral fat did not change consistently.

Blood ketones rose markedly, but to a variable extent, in all starved monkeys. The ketonemia

TABLE II

Serum lipids and blood ketones of monkeys

\begin{tabular}{|c|c|c|c|c|c|c|c|c|c|}
\hline \multirow{3}{*}{ Monkey } & \multirow{3}{*}{ Days } & \multicolumn{5}{|c|}{ Serum lipids } & \multirow{3}{*}{$\begin{array}{l}\text { Ketones } \\
\text { as } \\
\text { acetone }\end{array}$} & \multirow{3}{*}{$\begin{array}{c}\text { Total } \\
\text { proteins }\end{array}$} & \\
\hline & & \multicolumn{2}{|c|}{ Cholesterol } & \multirow{2}{*}{$\begin{array}{l}\text { Lipid } \\
\text { phos- } \\
\text { phorus }\end{array}$} & \multicolumn{2}{|c|}{ Fatty acids } & & & \\
\hline & & Total & Free & & Total & $\begin{array}{l}\text { Neutral } \\
\text { fat }\end{array}$ & & & \\
\hline 1545 & $\begin{array}{l}0 \\
3\end{array}$ & $\begin{array}{c}\text { mgm. per } \\
100 \text { cc. } \\
105 \\
155\end{array}$ & $\begin{array}{c}\text { mgm. per } \\
100 \text { cc. } \\
24 \\
30\end{array}$ & $\begin{array}{c}\text { mgm. per } \\
100 \text { cc. } \\
7.8 \\
10.7\end{array}$ & $\begin{array}{c}\text { m.eq. per } \\
1000 \text { cc. } \\
7.8 \\
10.7\end{array}$ & $\begin{array}{c}\text { m.eg. per } \\
1000 \text { cc. } \\
1.4 \\
0.8\end{array}$ & $\begin{array}{c}\text { mgm. per } \\
100 \text { cc. } \\
\text { whole } \\
\text { blood } \\
1.1^{*} \\
12.9\end{array}$ & $\begin{array}{c}\text { grams per } \\
100 \text { cc. } \\
\text { serum } \\
7.1 \\
7.7^{*}\end{array}$ & $\begin{array}{l}\text { Control study, eating normally but in } \\
\text { post-absorptive state } \\
\text { After } 84 \text { hours without food }\end{array}$ \\
\hline \multirow[t]{6}{*}{2156} & $\begin{array}{l}0 \\
5\end{array}$ & $\begin{array}{l}141 \\
134\end{array}$ & $\begin{array}{l}33 \\
30\end{array}$ & 8.7 & $\begin{array}{l}9.0 \\
8.9\end{array}$ & 1.1 & 0.9 & 7.6 & $\begin{array}{l}\text { Control, eating normally, but in post- } \\
\text { absorptive state }\end{array}$ \\
\hline & $\begin{array}{l}0 \\
2 \\
6\end{array}$ & $\begin{array}{l}157 \\
181 \\
225\end{array}$ & $\begin{array}{l}45 \\
46^{* *} \\
51\end{array}$ & $\begin{array}{l}8.8 \\
9.9\end{array}$ & $\begin{array}{r}9.0 \\
10.7 \\
10.6\end{array}$ & $\begin{array}{l}1.0 \\
2.3\end{array}$ & $\begin{array}{l}36.6^{*} \\
46.9\end{array}$ & $\begin{array}{l}7.2 \\
7.4\end{array}$ & $\begin{array}{l}\text { After } 2 \text { days without food } \\
\text { After } 6 \text { days without food }\end{array}$ \\
\hline & $\begin{array}{l}0 \\
7\end{array}$ & $\begin{array}{l}132 \\
176\end{array}$ & $\begin{array}{l}25 \\
46\end{array}$ & $\begin{array}{l}8.4 \\
8.1\end{array}$ & $\begin{array}{l}6.9 \\
8.6\end{array}$ & $\begin{array}{r}-0.8 \\
0.5\end{array}$ & $\begin{array}{l}0.7 \\
0.8\end{array}$ & & $\begin{array}{l}\text { Control study, eating normally } \\
\text { After } 1 \text { week of } 15 \text { grams of sucrose daily }\end{array}$ \\
\hline & $\begin{array}{l}\mathbf{0} \\
\mathbf{5}\end{array}$ & $\begin{array}{l}117 \\
220\end{array}$ & $\begin{array}{l}25 \\
45\end{array}$ & $\begin{array}{r}7.7 \\
10.4\end{array}$ & $\begin{array}{r}8.2 \\
13.2\end{array}$ & $\begin{array}{l}1.4 \\
2.6\end{array}$ & $\begin{array}{ll}1.0^{*} \\
58.8\end{array}$ & $\begin{array}{l}6.2 \\
7.1^{*}\end{array}$ & $\begin{array}{l}\text { Control, eating normally } \\
\text { After } 5 \text { days of starvation }\end{array}$ \\
\hline & $\begin{array}{l}0 \\
4 \\
8\end{array}$ & $\begin{array}{l}152 \\
197 \\
155\end{array}$ & $\begin{array}{l}38 \\
46 \\
39\end{array}$ & $\begin{array}{r}9.7 \\
10.5 \\
8.5\end{array}$ & $\begin{array}{r}9.3 \\
10.2 \\
9.6\end{array}$ & $\begin{array}{l}0.8 \\
0.5 \\
0.6\end{array}$ & $\begin{array}{l}0.9^{*} \\
5.2 \\
0.8\end{array}$ & $\begin{array}{l}6.6 \\
7.3 \\
7.2\end{array}$ & $\begin{array}{l}\text { Control, eating normally } \\
15 \text { grams sucrose daily for } 4 \text { days } \\
\mathbf{4 0} \text { grams sucrose daily for } 4 \text { days }\end{array}$ \\
\hline & $\begin{array}{l}0 \\
3 \\
7\end{array}$ & $\begin{array}{l}145 \\
157\end{array}$ & $\begin{array}{l}31 \\
39\end{array}$ & $\begin{array}{l}8.5 \\
9.2\end{array}$ & $\begin{array}{l}9.7 \\
9.5\end{array}$ & $\begin{array}{l}1.8 \\
1.1\end{array}$ & $\begin{array}{l}1.3 \\
4.3 \\
1.2\end{array}$ & $\begin{array}{l}6.4 \\
6.8\end{array}$ & $\begin{array}{l}\text { Control } \\
15 \text { grams sucrose daily } \\
40 \text { grams sucrose for } 4 \text { days }\end{array}$ \\
\hline \multirow[t]{3}{*}{1739} & $\begin{array}{l}0 \\
0 \\
0 \\
2 \\
6\end{array}$ & $\begin{array}{l}113 \\
119 \\
119 \\
172 \\
189\end{array}$ & $\begin{array}{l}30 \\
29 \\
32 \\
40 \\
46\end{array}$ & $\begin{array}{r}8.4 \\
7.2 \\
10.7 \\
9.2\end{array}$ & $\begin{array}{l}7.3 \\
6.9 \\
6.5 \\
9.5 \\
8.8\end{array}$ & $\begin{array}{r}0.3 \\
0.1 \\
-0.2 \\
-0.3\end{array}$ & $\begin{array}{r}0.8 \\
12.6 \\
9.7\end{array}$ & $\begin{array}{l}6.6 \\
6.7 \\
6.9 \\
6.4\end{array}$ & $\begin{array}{l}\text { Control, eating normally but in post- } \\
\text { absorptive state } \\
\text { After } 2 \text { days without food } \\
\text { After } 6 \text { days without food }\end{array}$ \\
\hline & $\begin{array}{l}0 \\
7\end{array}$ & $\begin{array}{l}124 \\
191\end{array}$ & $\begin{array}{l}29 \\
49\end{array}$ & $\begin{array}{l}7.5 \\
9.0\end{array}$ & $\begin{array}{l}6.6 \\
8.7\end{array}$ & $\begin{array}{l}-0.3 \\
-0.2\end{array}$ & $\begin{array}{l}0.6 \\
1.4\end{array}$ & 6.6 & $\begin{array}{l}\text { Control } \\
\text { After } 1 \text { week } 15 \text { grams sucrose daily }\end{array}$ \\
\hline & $\begin{array}{l}0 \\
4\end{array}$ & $\begin{array}{l}162 \\
171\end{array}$ & $\begin{array}{l}40 \\
44\end{array}$ & $\begin{array}{l}9.2 \\
9.2\end{array}$ & $\begin{array}{l}8.7 \\
9.8\end{array}$ & $\begin{array}{l}0.2 \\
1.2\end{array}$ & $\begin{array}{l}0.7^{*} \\
4.0\end{array}$ & $\begin{array}{l}6.0 \\
6.9\end{array}$ & $\begin{array}{l}\text { Control } \\
\text { After } 4 \text { days } 15 \text { grams sucrose daily }\end{array}$ \\
\hline 1567 & $\begin{array}{l}0 \\
0 \\
4 \\
7\end{array}$ & $\begin{array}{l}144 \\
134 \\
157 \\
107\end{array}$ & $\begin{array}{l}32 \\
39 \\
41 \\
31\end{array}$ & $\begin{array}{l}8.9 \\
9.1 \\
7.9 \\
7.4\end{array}$ & $\begin{array}{l}9.5 \\
9.2 \\
7.4 \\
7.7\end{array}$ & $\begin{array}{r}0.9 \\
1.3 \\
-0.1 \\
1.5\end{array}$ & $\begin{array}{l}1.6 \\
1.3 \\
2.8\end{array}$ & 7.2 & $\begin{array}{l}\text { Control } \\
\text { Control } \\
\text { After } 4 \text { days } 20 \text { grams sucrose daily } \\
\text { After } 3 \text { days of } 50 \text { grams sucrose daily }\end{array}$ \\
\hline
\end{tabular}

* One determination. 
usually was greatest in the animals that starved longest. No definite quantitative correlation between hyperlipemia and ketonemia could be established in these experiments.

Ingestion of 15 to 20 grams of sugar daily for from 3 to 7 days prevented the rise of lipid phosphorus, but not of cholesterol, and diminished ketonemia. When 40 to 50 grams of sugar were given daily, ketonemia was abolished and serum cholesterol fell to or below its initial concentration. This is distinctly less than the daily amounts of carbohydrate given to monkeys in their regular diets.

\section{Experiments with dogs}

Seven dogs were starved for from 4 to 14 days. The data are not presented in detail because there were no consistent changes of the serum lipids. In one animal, after 14 days, blood ketones rose to $1.5 \mathrm{mgm}$. per cent.

\section{DISCUSSION}

It has been rather generally stated that starvation is attended by hyperlipemia in which neutral fat is particularly involved. Evidence for these statements is, however, largely inferential, based on studies of diabetes uncontrolled by insulin and on the well-established fact that the lipids of the liver increase in starvation. In the present studies, a slight, but significant, increase of serum lipids has been demonstrated in normal men during starvation. A more pronounced hyperlipemia was observed in monkeys. The serum lipids of the dog were unaffected by starvation. In all species, neutral fat was only slightly altered. In both man and monkey, the lipid increment consisted of cholesterol and phospholipid, the former predominating.

The human experiments are somewhat complicated by the presence of hemoconcentration which regularly attends starvation and was exaggerated in the majority of these experiments by water deprivation. It has been shown by Man and Peters (29) that, when acute hemoconcentration is induced by prolonged maintenance of the erect posture, serum lipids and proteins rise proportionally. Evidence has also been adduced that the hyperlipemia of diabetic acidosis may be referable in part to hemoconcentration (30). During recovery after the acute phase of this condition, serum lipids and proteins parallel one another in their descent. Hemoconcentration may have been responsible for some part, but not all, of the increases of lipids in the human starvation experiments. Cholesterol rose proportionally more than the serum proteins. In addition, ingestion of carbohydrate in Experiment I inhibited hyperlipemia without greatly mitigating dehydration, while the lipids of $\mathrm{Br}, \mathrm{Bl}$, and $\mathrm{Fl}$ (Experiment IV) rose, although the subjects were allowed to drink water ad libitum. In the monkeys, lipids rose so much more than proteins that the reality of the hyperlipemia cannot be questioned.

The general concept that the hyperlipemia of starvation arises merely from the rapid mobilization of fat seems hardly tenable in view of these observations. Already there is a wealth of evidence that the concentrations of lipids in the serum are little influenced by the quantity of fat in the metabolism mixture. After fatty meals, there is a transient hyperlipemia, affecting chiefly neutral fat (31 to 36). On the other hand, the serum lipids-and especially cholesterol-of a given individual remain remarkably constant throughout a day and over long periods despite variations of diet $(24,25,34,35,37$ to 39$)$. Starvation lipemia must, therefore, be attributed to a change in the character, rather than the quantity of fat metabolism.

The most obvious phenomenon with which to connect it is ketosis. The dog, inured to a carbohydrate-free diet, does not change the character of its metabolism radically with starvation. It also develops neither ketosis (10) nor lipemia $(1$ to $5,8,9)$. In the diabetic dog, however, both ketosis (40 to 42 ) and lipemia (43 to 47) occur. The human male, when starved, exhibits mild ketosis and a comparably slight lipemia. The two phenomena tend to parallel one another. The monkey is somewhat more susceptible to ketosis $(42,48)$ and has a proportionallý greater lipemia.

In both man and monkey, lipemia can be abolished by the administration of quantities of carbohydrate altogether too small to alter radically the quantities of fat oxidized, but large enough to reduce ketosis to minimal proportions. Reduction of ketosis by administration of carbohydrate has been repeatedly reported $(11,15,41)$. 
Transfer from a mixed diet to 100 grams of carbohydrate, in the case of an adult male, for example, which must involve the consumption of at least 1200 additional Calories from fat per day, had no appreciable effect on serum lipids. Nevertheless, removal of 400 Calories in the form of sugar, thereby provoking ketosis, elicited distinct hyperlipemia. The fatty meals given by Man and Gildea (36) contained as much as 2500 Calories of fat. In the monkey, also, amounts of carbohydrate too small to reduce considerably the quantities of fat metabolized, but large enough to mitigate ketosis, reduced hyperlipemia.

Greater ketosis and lipemia might have been demonstrable in women and in children than in adult males. Deuel and Gulick found greater ketonemia in fasting women than in men (49). McQuarrie, Husted, and Bloor (50) have reported striking elevations of cholesterol, phospholipids, and fatty acids in the serum of epileptic children receiving ketogenic diets. The lipids fell when enough carbohydrate was given to eliminate ketosis. Hypercholesterolemia has also been reported by Tolstoi and his associates ( 51 , 52) in an adult male who subsisted for prolonged periods on diets consisting solely of fat and protein. This is at variance with the report of Corcoran and Rabinowitch (53) of diminished rather than elevated serum cholesterol and phospholipids in Eskimos. The latter, however, did not have ketonemia, while Tolstoi's subjects did.

In the acidosis of human diabetes, in which both ketosis and hyperlipemia attain a severity never reached in starvation, neutral fat is usually affected as much or more than are cholesterol and lipid phosphorus (30). In this respect, for some reason, the lipemia differs from that of starvation. The distinction is not, however, an absolute one. Although cholesterol and lipid phosphorus are always elevated, neutral fat sometimes escapes. This is illustrated by comparison of the first 3 and last 3 cases in Table III (cases and treatment described previously (30)). What determines the participation of neutral fat is not clear. It may be the severity of the ketosis or the nutritive state of the patient; but it is impossible to establish a clear correlation with either of these features from the data now available. The consistent rises of cholesterol and lipid phosphorus, in contrast to the capricious
TABLE III

Serum lipids in diabetic acidosis of humans

\begin{tabular}{|c|c|c|c|c|c|c|}
\hline \multirow[b]{2}{*}{$\begin{array}{c}\text { Case } \\
\text { number, } \\
\text { sex }\end{array}$} & \multirow[b]{2}{*}{ Days, Hours } & \multirow[b]{2}{*}{$\begin{array}{l}\text { Blood } \\
\text { sugar }\end{array}$} & \multicolumn{4}{|c|}{ Serum } \\
\hline & & & $\begin{array}{l}\text { Carbon } \\
\text { dioxide }\end{array}$ & Choles- & $\begin{array}{c}\text { Lipid } \\
\text { phos- } \\
\text { phorus }\end{array}$ & $\begin{array}{c}\text { Fatty } \\
\text { acids of } \\
\text { neutral } \\
\text { fat }\end{array}$ \\
\hline $\begin{array}{c}\text { A700 } \\
\text { F }\end{array}$ & $\begin{array}{r}3 \\
1,11 \\
3,11 \\
5,11\end{array}$ & $\begin{array}{c}\text { per } \\
100 \text { cc. } \\
686 \\
480 \\
375 \\
530 \\
360\end{array}$ & $\begin{array}{c}\text { volumes } \\
\text { per } \\
\text { cent }\end{array}$ & $\begin{array}{c}\text { mgm. } \\
\text { per } \\
100 \text { cc. } \\
673 \\
680 \\
456 \\
559 \\
503\end{array}$ & $\begin{array}{c}\text { mgm. } \\
\text { per } \\
100 \text { cc. } \\
18.9 \\
16.3 \\
12.7 \\
13.3 \\
12.8\end{array}$ & $\begin{array}{c}\text { m.eq. } \\
\text { per } \\
1000 \text { cc. } \\
8.2 \\
5.6 \\
6.4 \\
4.3 \\
5.7\end{array}$ \\
\hline$\underset{F}{A 30940}$ & 7 & $\begin{array}{r}1080 \\
94\end{array}$ & $\begin{array}{l}26.9 \\
46.4\end{array}$ & $\begin{array}{l}157 \\
110\end{array}$ & $\begin{array}{l}8.8 \\
5.7\end{array}$ & $\begin{array}{l}3.1 \\
3.1\end{array}$ \\
\hline$\underset{F}{A 30929}$ & 12 & $\begin{array}{l}649 \\
474\end{array}$ & 27.1 & $\begin{array}{l}454 \\
452\end{array}$ & $\begin{array}{l}13.4 \\
12.8\end{array}$ & $\begin{array}{l}3.9 \\
4.4\end{array}$ \\
\hline$\underset{M}{A 25652}$ & 3 & $\begin{array}{l}418 \\
492\end{array}$ & 11.6 & $\begin{array}{l}490 \\
212\end{array}$ & $\begin{array}{l}19.9 \\
10.6\end{array}$ & $\begin{array}{l}14.4 \\
12.0\end{array}$ \\
\hline A5815 & $\begin{array}{c}5 \\
9 \frac{1}{2} \\
14\end{array}$ & $\begin{array}{r}713 \\
476 \\
168 \\
60 \\
267 \\
486 \\
275\end{array}$ & $\begin{array}{l}11.4 \\
29.3 \\
41.6 \\
42.7 \\
44.9 \\
43.2\end{array}$ & $\begin{array}{l}304 \\
281 \\
213 \\
208 \\
203 \\
190 \\
209\end{array}$ & $\begin{array}{r}20.9 \\
14.5 \\
10.0 \\
9.5 \\
10.1 \\
9.5 \\
11.4\end{array}$ & $\begin{array}{r}30.1 \\
12.0 \\
8.2 \\
5.5 \\
6.3 \\
7.2 \\
7.1\end{array}$ \\
\hline$\underset{F}{29923}$ & $\begin{array}{l}1 \\
2\end{array}$ & 716 & $\begin{array}{l}11.7 \\
32.1 \\
34.5\end{array}$ & $\begin{array}{l}233 \\
161 \\
117\end{array}$ & $\begin{array}{r}16.3 \\
8.5 \\
6.2\end{array}$ & $\begin{array}{r}32.3 \\
4.2 \\
4.0\end{array}$ \\
\hline
\end{tabular}

action of neutral fat, are common to both starvation and diabetic acidosis.

The lipid disturbances of acute starvation contrast sharply with those of chronic undernutrition. In the latter, the serum lipids are characteristically reduced $(54,55)$. Administration of carbohydrate in starvation causes a rapid decline of the lipids; during recovery from malnutrition, the lipids rise from subnormal to normal concentrations. Nevertheless, there is reason to believe that starvation will cause the lipids of a chronically malnourished patient to rise as do those of normal subjects. During acidosis in malnourished diabetics, Man and Peters (30) observed normal or high concentrations of lipids that fell below normal on recovery. An illustration is found in Case A30940 of Table III. The course of the serum lipids during starvation in a malnourished patient without diabetes is illustrated in Figure 5. The woman had an inoperable gastric carcinoma causing pyloric obstruction. On admission, she had taken little food for many 


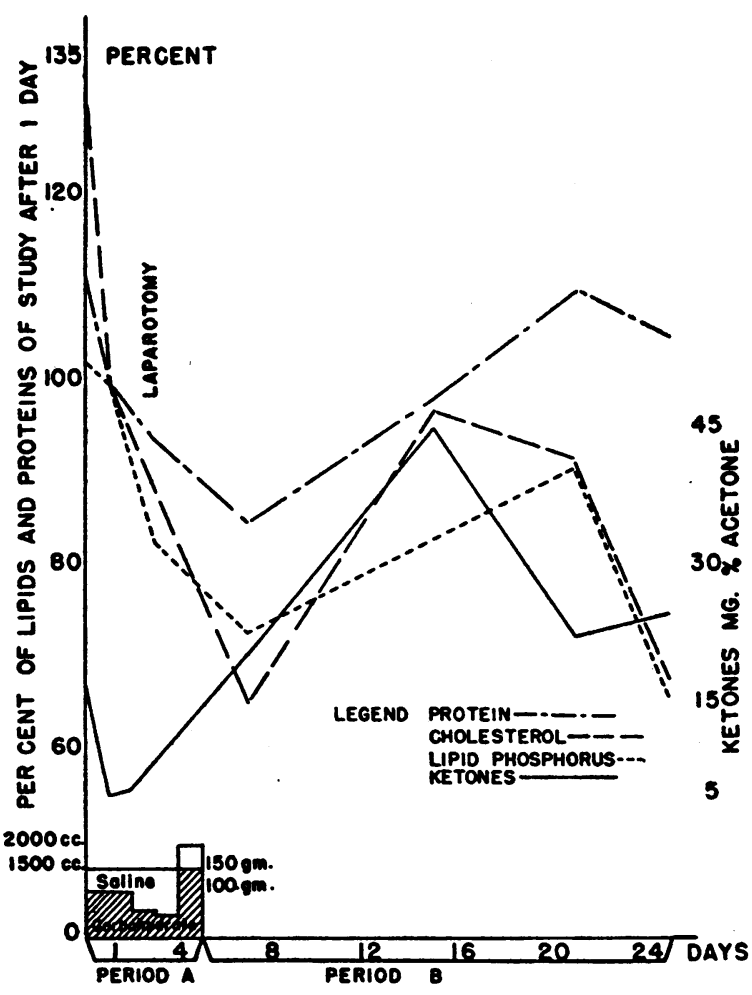

Fig. 5. Blood Ketones and Serum Proteins, Lipid Phosphorus and Total Cholesterol of a Patient with an INOPERable, OBSTRUCTING GASTRIC Carcinoma

To represent proportionately the changes in serum lipid fractions and proteins, the values on the first morning after parenteral fluid and glucose have been selected as 100 per cent. These relative values can be translated into absolute terms from the following data: at 100 per cent, protein equals 5.58 per cent, cholesterol $163 \mathrm{mgm}$. per cent, and lipid phosphorus $10.4 \mathrm{mgm}$. per cent. Blood ketones are presented in absolute terms.

weeks and had vomited everything for 4 days or more. During the first few days (period A of Figure 5), while she received parenteral saline and glucose, all the serum lipid fractions fell considerably and progressively. Cholesterol diminished more than lipid phosphorus did, finally falling below the normal range. Serum proteins also declined, but relatively less than the lipids. Blood ketones diminished within 24 hours, but never to normal values. With resumption of starvation (period B), blood ketones and lipids again rose for a time. The initial study in period $\mathrm{B}$ is difficult to evaluate because no blood study was obtained soon after carbohydrate ad- ministration was stopped. As starvation was prolonged and wasting became extreme, both ketones and lipids gradually decreased again.

These experiments and the other scanty data which can be found in the literature strongly suggest that the hyperlipemia of carbohydrate starvation is not simply an indication that fat is being burned more rapidly, but that a larger proportion of fatty acids is being converted to ketone bodies in the process of combustion. There is ample evidence that the quantity of fat in the metabolism mixture can be varied greatly without any demonstrable variation of serum lipids so long as it is burned directly by the tissues and no unusual amounts of ketone bodies are formed. When, however, ketogenesis exceeds certain limits, hyperlipemia appears. In this, cholesterol and phospholipids seem to play a major role, presumably serving as vehicles to convey the fatty acids to the liver. It may be that they are required to facilitate the entrance of the fatty acids into the liver and participate in the reactions by which ketone bodies are formed. It is well recognized that the hepatic metabolism of fat is greatly influenced by phospholipids and cholesterol.

\section{SUMMARY AND CONCLUSIONS}

A study has been made of the serum lipids and blood ketones of adult human males, dogs, and monkeys during starvation.

Dogs developed no appreciable ketosis and no hyperlipemia.

In men, blood ketones increased progressively throughout the periods of starvation, which varied from 2 to 6 days. Serum cholesterol rose slightly, but unequivocally, as fasting was prolonged; lipid phosphorus rose perceptibly; neutral fat changes were equivocal.

In monkeys both blood ketones and serum lipids rose more rapidly and further than they did in men. Again cholesterol was most affected, while neutral fat did not change appreciably.

In both man and monkey, the hyperlipemia was abolished by administration of sufficient carbohydrate to mitigate or to extinguish the ketosis.

From this and other evidence, it is suggested that the hyperlipemia of carbohydrate starvation arises not merely because a larger quantity of 
fat is being utilized, but because an unusually large amount of fat is being converted to ketone bodies.

\section{BIBLIOGRAPHY}

1. Daddi, L., Sur les modifications du poids de l'extrait éthéré du sang durant le jeûne de longue durée. Arch. ital. de biol., 1898, 30, 439.

2. Bloor, W. R., Studies on blood fat. I. Variations in the fat content of the blood under approximately normal conditions. J. Biol. Chem., 1914, 19, 1.

3. Bang, I., Uber Lipämie II. Biochem. Ztschr., 1918, 91, 104.

4. Wendt, H., Lipoidstoffwechselstudien am Hungertier. Klin. Wchnschr., 1928, 7, 2183.

5. Terroine, E. F., Physiologie des substances grasses et lipoidiques. Annales des Sciences Naturelles. Zoologie., 1920, Series 10, Vol. 4.

6. Shope, R. E., Sugar and cholesterol in the blood serum as related to fasting. J. Biol. Chem., 1927, 75, 101.

7. Sure, B., Kik, M. C., and Church, A. E., The influence of fasting on the concentration of blood lipids in the albino rat. J. Biol. Chem., 1933, 103, 417.

8. Entenman, C., Changus, G. W., Gibbs, G. E., and Chaikoff, I. L., The response of lipid metabolism to alterations in nutritional state. I. The effects of fasting and chronic undernutrition upon the postabsorptive level of the blood lipids. J. Biol. Chem., 1940, $134,59$.

9. Bloor, W. R., Biochemistry of the Fatty Acids and Their Compounds, the Lipids. Reinhold Publishing Corp., New York City, 1943.

10. Crandall, L. A., Jr., A method for acetone bodies in blood, applicable to the determination of small amounts of mercury. J. Biol. Chem., 1940, 133, 539.

11. Crandall, L. A., Jr., A comparison of ketosis in man and dog. J. Biol. Chem., 1941, 138, 123.

12. Drury, D. R., Wick, A. N., and MacKay, E. M., The action of exercise on ketosis. Am. J. Physiol., 1941, 134, 761.

13. Mirsky, I. A., Nelson, N., and Elgart, S., Diabetic acidosis and coma in the monkey. Science, 1941, 93, 576.

14. Mirsky, I. A., Nelson, N., Grayman, I., and Elgart, S., Pancreatic diabetes in the monkey. Endocrinology, 1942, 31, 264.

15. Somogyi, M., Effects of glucose feeding upon the ketonemia in healthy man. J. Biol. Chem., 1942, $145,575$.

16. Winkler, A. W., Danowski, T. S., Elkinton, J. R., and Peters, J. P., Electrolyte and fluid studies during water deprivation and starvation in human subjects, and the effect of ingestion of fish, of carbohydrate, and of salt solutions. J. Clin. Invest., 1944, 23, 807.

17. Lozner, E. L., Winkler, A. W., Taylor, F. H. L., and Peters, J. P., The intravenous glucose tolerance test. J. Clin. Invest., 1941, 20, 507.

18. Man, E. B., and Gildea, E. F., A modification of the Stoddard and Drury titrimetric method for the determination of the fatty acids in blood serum. J. Biol. Chem., 1932, 99, 43.

19. Man, E. B., and Gildea, E. F., Notes on the extraction and saponification of lipids from blood and blood serum. J. Biol. Chem., 1937, 122, 77.

20. Man, E. B., and Peters, J. P., Gravimetric determination of serum cholesterol adapted to the Man and Gildea fatty acid method, with a note on the estimation of lipoid phosphorus. J. Biol. Chem., 1933, 101, 685.

21. Man, E. B., A note on the stability and quantitative determination of phosphatides. J. Biol. Chem., 1937, 117, 183.

22. Bogdanovitch, S. B., and Man, E. B., The effects of castration, theelin, testosterone and antuitrin-S on the lipoids of blood, liver and muscle of guinea pigs. Am. J. Physiol., 1938, 122, 73.

23. Bruckman, F. S., D'Esopo, L. M., and Peters, J. P., The plasma proteins in relation to blood hydration. IV. Malnutrition and serum proteins. J. Clin. Invest., 1930, 8, 577.

24. Peters, J. P., and Man, E. B., The interrelations of serum lipids in normal persons. J. Clin. Invest., 1943, 22, 707.

25. Man, E. B., and Gildea, E. F., Variations in lipemia of normal subjects. J. Biol. Chem., 1937, 119, 769.

26. Weichselbaum, T. E., and Somogyi, M., A method for the determination of small amounts of ketone bodies. J. Biol. Chem., 1941, 140, 5.

27. Sperry, W. M., The concentration of total cholesterol in the blood serum. J. Biol. Chem., 1937, 117, 391.

28. Sperry, W. M., The relationship between total and free cholesterol in human blood serum. J. Biol. Chem., 1936, 114, 125.

29. Man, E. B., and Peters, J. P., Permeability of capillaries to plasma lipoids. J. Clin. Invest., 1933, 12, 1031.

30. Man, E. B., and Peters, J. P., Lipoids of serum in diabetic acidosis. J. Clin. Invest., 1934, 13, 237.

31. Bang, I., Über Lipämie III. Biochem. Ztschr., 1918, 91, 111.

32. Bang, I., Über Cholesterinämie. Biochem. Ztschr., 1918, 91, 122.

33. Hiller, A., Linder, G. C., Lundsgaard, C., and Van Slyke, D. D., Fat metabolism in nephritis. J. Exper. Med., 1924, 39, 931.

34. Bruger, M., and Somach, I., The diurnal variations of the cholesterol content of the blood. J. Biol. Chem., 1932, 97, 23.

35. Oppenheim, E., and Bruger, M., The effect of a high-fat test meal on blood cholesterol in normal and obese individuals. Am. J. M. Sc., 1943, 205, 77.

36. Man, E. B., and Gildea, E. F., The effect of the ingestion of a large amount of fat and of a balanced meal on the blood lipids of normal man. J. Biol. Chem., 1932, 99, 61.

37. Turner, K. B., and Steiner, A., A long term study of the variation of serum cholesterol in man. J. Clin. Invest., 1939, 18, 45. 
38. Boyd, E. M., Diurnal variations in plasma lipids. J. Biol. Chem., 1935, 110, 61.

39. Brun, G., Changes in the Lipide Contents of Serum in Patients with Manic-Depressive Psychosis. $\mathrm{H}$. K. Lewis, London, 1940.

40. Chaikoff, I. L., Macleod, J. J. R., Markowitz, J., and Simpson, W. W., Observations on depancreatized dogs before and after the withdrawal of insulin. Am. J. Physiol., 1925, 74, 36.

41. Mirsky, I. A., Heiman, J. D., and Broh-Kahn, R. H., The antiketogenic action of glucose in the absence of insulin. Am. J. Physiol., 1937, 118, 290.

42. Friedemann, T. E., Ketosis in primates. II. The ratio of $\beta$-hydroxybutyric acid to acetoacetic acid in blood and urine. J. Biol. Chem., 1942, 142, 635.

43. Seo, Y., Utber das Vorkommen von Lipämie und über die Menge der Lipoidsubstanzen in Blut und Leber beim Pankreasdiabetes. Arch. f. exper. Path. u. Pharmakol., 1909, 61, 1.

44. Allen, F. M., Experimental studies in diabetes. IV. Lipemia. 2. The production of diabetic lipemia in animals and observations on some possible etiologic factors. J. Metab. Research, 1922, 2, 219.

45. Wishart, M. B., Experimental studies in diabetes. IV. Lipemia. 1. Analyses of blood lipoids in diabetic animals and patients. J. Metab. Research, 1922, 2, 199.

46. Nitzescu, I. I., Popsecu-Inotesti, C., and Cadariu, I., L'insuline et la cholestérinémie dans le diabète expérimentale. Compt. rend. soc. de Biol., 1924, 90, 538.

47. Bloor, W. R., Gillette, E. M., and James, M. S., Fat metabolism in diabetes. I. The blood lipids in experimental diabetes. J. Biol. Chem., 1927, 75, 61 .

48. Friedemann, T. E., The fasting ketosis of monkeys. J. Biol. Chem., 1934, 105, 335.

49. Deuel, H. J., Jr., and Gulick, M., Studies on ketosis. I. The sexual variation in starvation ketosis. J. Biol. Chem., 1932, 96, 25.

50. McQuarrie, I., Husted, C., and Bloor, W. R., The lipids of the blood plasma in epilepsy. II. Variations of lipids in relation to occurrence of seizures. J. Clin. Invest., 1933, 12, 255.

51. Tolstoi, E., The effect of an exclusive meat diet on the chemical constituents of the blood. J. Biol. Chem., 1929, 83, 753.

52. McClellan, W. S., and DuBois, E. F., Clinical calorimetry. XLV. Prolonged meat diets with a study of kidney function and ketosis. J. Biol. Chem., 1930, 87, 651.

53. Corcoran, A. C., and Rabinowitch, I. M., A study of the blood lipoids and blood protein in Canadian eastern Arctic Eskimos. Biochem. J., 1937, 31, 343.

54. Man, E. B., and Gildea, E. F., Serum lipoids in malnutrition. J. Clin. Invest., 1936, 15, 203.

55. Hodges, R. G., Sperry, W. M., and Andersen, D. H., Serum cholesterol values for infants and children. Am. J. Dis. Child., 1943, 65, 858. 